\title{
Matrices progresivas de Raven: punto de corte para preescolares 4 - 6 años
}

\author{
Yaser Ramírez Benítez ${ }^{1}$, Rodneys Mauricio Jiménez-Morales ${ }^{2}$, Miriela Díaz Bringas ${ }^{3}$ \\ ${ }^{1}$ Universidad de Cienfuegos "Carlos R Rodríguez". Centro Docente de Rehabilitación del Neurodesarrollo. \\ ${ }^{2}$ Universidad de Ciencias Médicas Dr. Faustino Pérez Hernández. Hospital Provincial de Rehabilitación de \\ Sancti Spíritus, Cuba. \\ ${ }^{3}$ UCMM "Juan Guiteras Gener". Servicio de Neuropsicología del Centro Docente de Rehabilitación del \\ Neurodesarrollo "Rosa Luxemburgo". Cárdenas, Cuba.
}

La correspondencia relacionada con este artículo debe enviarse a: Yaser Ramírez Benítez. Universidad de Cienfuegos “Carlos Rafael Rodríguez”, Carretera Central, 4 Caminos, Cienfuegos www.ucf.edu.cu Tel. 43 549 608. Dirección: yaser@citmacfg.cu

\begin{abstract}
Resumen. Se realizó un estudio descriptivo - correlacional en varios centros educativos de las provincias de Cienfuegos y Matanzas para determinar un punto de corte del niño preescolar $4-6$ años. Se utilizó dos muestras: muestra de normalización (N1) y muestra de validación (N2). N1 quedó conformada por 406 niños: 108 con 4 años, 102 con 5 años y 196 con 6 años. N2 quedó conformada por 130 niños: 30 con 4 años, 50 con 5 años y 50 con 6 años. Se utilizó el método Curva Receptor- Operador para seleccionar el punto de corte. La validez de la prueba se obtuvo a través de la correlación con dos tareas de razonamiento (Semejanzas - Diferencias y Operaciones numéricas). El Alfa de Cronbach indicó la confiabilidad de la prueba. La prueba presenta propiedades psicométricas adecuadas para aplicarse en la edad preescolar. Los puntos de corte seleccionados mostraron valores apropiados para detectar el riesgo intelectual en la etapa preescolar.

Palabras clave: Raven, inteligencia, coeficiente intelectual, razonamiento, preescolar
\end{abstract}

\begin{abstract}
A descriptive study was conducted - correlational in several schools in the provinces of Cienfuegos and Matanzas to determine a cut-off of the preschool child 4 to 6 years. Standardization sample (N1) and validation sample (N2): two samples were used. N1 was composed of 406 children: 1084 years 1025 years 1966 years. N2 was composed of 130 children: 30 to 4 years, 50 with 5 years and 50 to 6 years. The receiver-operator curve method was used to select the cut. The validity of the test was obtained through correlation with two reasoning tasks (Similarities - Differences and numerical operations). The Cronbach's alpha indicated the reliability of the test. The test presents suitable for application in preschool psychometric properties. The cutoffs selected showed appropriate values to detect intellectual risk in preschool.
\end{abstract}

Keywords: Raven, intelligent coefficient, preschool, intelligence, preschool

\section{INTRODUCCIÓN}

Las Matrices Progresivas Coloreadas de Raven es un instrumento apropiado para obtener un perfil intelectual no verbal. La tarea es de rápida y fácil aplicación, por lo cual permite realizar investigaciones en grandes poblaciones en poco tiempo y con menos recursos humanos y materiales.

La preferencia y el atractivo del test no solo están en su aplicación, también en el resultado que muestra. El instrumento es por excelencia una medida de inteligencia fluida 
(Gf), por tanto la solución no depende de conocimientos previos adquiridos en la educación, más bien se apoya en conocimiento universales simples (arriba / abajo, derecha / izquierda, poco / mucho). Además, Gf es una habilidad que tiene una fuerte relación con el aprendizaje en los primeros años de vida, pues el niño con altas puntuaciones en tareas Gf tiene posibilidades de obtener altas puntuaciones en habilidades académicas (Manrique Millones, Flores-Mendoza, \& Millones Rivalles, 2015; Ritchie, Bates, \& Plomin, 2015; Tong \& Fu, 2013). Asimismo, muestra una relación significativa con la maduración del sistema nervioso central, esencialmente con la producción de la dopamina y el desarrollo de las regiones frontoparietales (Schlagenhauf et al., 2013; Yuan et al., 2012).

En este sentido, la prueba Raven es utilizada en niños por tres razones fundamentales: primero, es un test de rápida y fácil aplicación. Segundo, puede ser una opción para poblaciones infantiles con alteraciones neurológicas con o sin alteraciones del habla (sordera, hipoacusia, afasias, autismo). Tercero, por la relación con las habilidades escolares (Barkl, Porter, \& Ginns, 2012; Desco et al., 2011; Primi, Ferrão, \& Almeida, 2010; Van Bergen et al., 2013), así como la memoria de trabajo (Leeuwen, Van den Berg, Hoekstra, \& Boomsma, 2007; Luo, Thompson, \& Detterman, 2006; Zhao, Wang, Liu, \& Zhou, 2011).

De este modo, los investigadores utilizan con frecuencia el instrumento para detectan el riesgo intelectual en grandes poblaciones, pues las pruebas tradicionales (Escala de Wechsler, Escala de Stanford Binet, Test de Inteligencia de Kaufman), pese a su gran importancia y utilidad, son extensas y complicadas.

El instrumento Raven surgen como una oportunidad para responder a las demandas del investigador, sin embargo no existen normas cubanas del instrumento, así como un punto de corte para detectar el riesgo intelectual en la población preescolar.

En la etapa preescolar, la experiencia social tiene una fuerte influencia sobre el desarrollo cerebral y conductual del niño, por tanto detectar puntuaciones bajas en la prueba Raven es una alerta para estimular el razonamiento analógico y en consecuencia preparar al niño para la próxima etapa escolar.

En el presente estudio, considerando la importancia de identificar el riesgo intelectual en la etapa preescolar, pretende determinar un punto de corte del test de Raven para el niño preescolar 4 - 6 años, así como determinar las propiedades psicométricas del test. 


\section{MÉTODO}

\section{Participantes}

Se realizó un diseño transversal con estudio descriptivo correlacional donde participaron dos muestras independientes.

Muestra de normalización (N1): Participaron 406 niños con 4,5 y 6,11 años asignados de forma aleatoria. La selección se hizo durante todo el año 2012 en las Círculos infantiles y Escuelas Primarias de Matanzas y Cienfuegos, Cuba (Tabla 1).

Tabla 1. Muestra de normalización distribuida por edades y sexo

\begin{tabular}{lrrrrr}
\hline \multirow{2}{*}{ Edad } & Total & \multicolumn{2}{c}{ Género } & \multicolumn{2}{c}{ Edad } \\
& & & & & \\
\cline { 2 - 6 } & & \multicolumn{1}{c}{ F } & \multicolumn{1}{c}{ M } & Media & DE \\
\hline 4.5 años & 108 & 51 & 63 & 4.6 & 0.19 \\
\hline 5 años & 102 & 49 & 53 & 5.1 & 0.51 \\
\hline 6 años & 198 & 95 & 103 & 6.1 & 0.28 \\
\hline Total & 406 & 195 & 219 & 5.5 & 0.73 \\
\hline
\end{tabular}

Muestra de validación (N2): Participaron 130 niños entre 4,5 años y 6,11 años seleccionados según criterios. Criterios de inclusión: niños entre $4-6$ años que acudieron a la consulta de neuropsicología en el Centro Docente de Rehabilitación del Neurodesarrollo, Cárdenas, Matanzas en el año 2012. Criterios de exclusión: niños entre $4-6$ años con una discapacidad intelectual severa y con alteraciones sensomotrices que impidan la aplicación del instrumento (Tabla 2).

Tabla 2. Muestra de validación distribuida por edades y sexo

\begin{tabular}{|c|c|c|c|c|c|}
\hline \multirow{2}{*}{ Edad } & \multirow[t]{2}{*}{ Total } & \multicolumn{2}{|c|}{ Género } & \multicolumn{2}{|c|}{ Edad } \\
\hline & & $\mathbf{F}$ & $\mathbf{M}$ & Media & DE \\
\hline 4,5 años & 30 & 14 & 16 & 4.4 & 0.21 \\
\hline 5 años & 50 & 25 & 25 & 5.3 & 0.33 \\
\hline 6 años & 50 & 23 & 27 & 6.4 & 0.28 \\
\hline Total & 130 & 62 & 68 & 5.4 & 0.81 \\
\hline
\end{tabular}

\section{Instrumentos}

Prueba de Inteligencia Matrices Progresivas Coloreada de Raven. Está constituida 
por 36 problemas, que se presentan en un cuaderno de papel. Cada uno de ellos consiste en una figura incompleta que el niño debe completar. Se presentan seis alternativas de respuesta posibles, una de ellas es la respuesta correcta y el resto son distractores. La prueba está dividida en tres series de doce problemas cada una: A, Ab y B. La complejidad se incrementa progresivamente a lo largo de la prueba y a lo interno de las series, tal que, A1 es el ítem más fácil y $\mathrm{B} 12$ el más difícil, pero $\mathrm{A} 12$ es más difícil que $\mathrm{B} 1$. Las funciones cognitivas que se examinan principalmente son: percepción de tamaños, percepción de orientación en el espacio, en una dirección y en dos direcciones simultáneamente, aprehensión de figuras discretas especialmente relacionadas con un todo (Series A y Ab), análisis de un todo en sus componentes, capacidad de concebir figuras correlativas y deducción de relaciones (Serie B).

Por cada respuesta correcta se otorga un punto y se recoge una puntuación total en la ejecución del test.

Batería neuropsicológica Luria Inicial. El instrumento evalúa cuatro dominios de la cognición para niños de 4 a 6 años: Ejecutivo, lingüístico, rapidez y memoria e incluye una prueba de lateralización manual (Manga \& Ramos, 2006). Está conformada por catorce tests donde los subtest "Semejanzas - diferencias" y “Operaciones numéricas” son tareas de razonamiento.

\section{Análisis de datos}

Descripción estadística. Se utilizó el SSPS y el Statistica para realizar los análisis estadísticos. Se determinó la media y la desviación estándar de la prueba en cada edad. Se aplicó la prueba t Students para conocer la relación que existía entre la muestra N1 vs N2.

Propiedades psicométricas. La fiabilidad del instrumento se obtuvo a través del método de bipartición (dividir el resultado en dos mitades). El procedimiento se realizó separando los ítems correspondientes a las posiciones pares e impares, para luego aplicar el Alfa de Cronbach por edades y de manera general. Los valores del alfa por encima de .70 fueron clasificados como buenos, los valores entre .80 - .90 fueron clasificados como adecuados y los valores por encima de .90 fueron superiores. La validez se obtuvo mediante un análisis de correlación entre el test de Raven y las subpruebas de razonamiento "Semejanzas - Diferencias" y "Operaciones numéricas".

Punto de corte. Para obtener el punto de corte se utilizó la curva receptor-operador 
(ROC). La interpretación de la curva se realizó de la siguiente manera: Entre 0,5 y 0,7 indican baja exactitud para clasificar a los niños de positivo y negativo; entre 0,7 y 0,9 pueden ser útiles para algunos propósitos y valores mayor de 0,9 indica alta exactitud (Swets, 1988). La validez del punto de corte se realizó en la muestra N2 usando como regla de oro las normas mexicanas del Raven (Raven, 2004).

\section{RESULTADOS}

\section{Descripción estadística}

La media mostró diferencias entre los grupos de edades en la muestra N1 como se muestra en la tabla 3. La prueba t reveló diferencias significativas entre la muestra N1 y N2 (Tabla 3).

Tabla 3. Estadísticos descriptivos de N1, prueba $\mathrm{t}$ de diferencias entre N1 y N2 y correlaciones entre el Raven y los subtests de razonamiento

\begin{tabular}{cccc}
\hline Edades & Media & DE & Prueba T (N1 vs N2) \\
\hline 4 años N1 $=108$ & 14.56 & 1.04 & .03 \\
\hline 5 años N1 $=102$ & 15.49 & 1.44 & .001 \\
\hline 6 años N1 $=196$ & 16.30 & 1.77 & .001 \\
\hline & Validez y fiabilidad & & Alfa de Cronbach \\
\hline
\end{tabular}

Semejanzas - Diferencias Operaciones numéricas

\begin{tabular}{cccc}
\hline 4 años & $.72^{* *}$ & $.63^{* *}$ & .82 \\
\hline 5 años & $.73^{* *}$ & $.74^{* *}$ & .89 \\
\hline 6 años & $.58^{* *}$ & $.58^{* *}$ & .91 \\
\hline Total & $.70^{* *}$ & $.69^{* *}$ & .86 \\
\hline
\end{tabular}

\section{Propiedades psicométricas}

\section{Validez}

La correlación entre el test de Raven y el dominio ejecutivo de la batería Luria Inicial fueron adecuadas: Semejanzas - Diferencias $\left(.70^{* *}\right)$ y Operaciones numéricas $(.69 * *)$ (Tabla 3). 


\section{Confiabilidad}

El alfa de Cronbach fue adecuada en toda la muestra (.86), a los 4 años (.82) y a los 5 años (.89), mientras que a los 6 años fue superior (.91) (Tabla 3).

\section{Punto de corte}

A los 4 años, 15 fue el punto de corte con mejor área debajo de la curva (.70). La sensibilidad fue de .76 y la especificidad de 1 . A los 5 años, 17 fue el punto de corte con mejor área debajo de la curva (.76). La sensibilidad fue 1 y la especificidad de .28. A los 6 años, 18 fue el punto de corte con mejor área debajo de la curva (.67). La sensibilidad fue de .87 y la especificidad de 1 . (Tabla 4).

Tabla 4. Área debajo de la Curva en la prueba Raven, Semejanzas - Diferencias y Operaciones numéricas. Indicadores de validez según el punto de corte seleccionado

\begin{tabular}{cccc}
\hline \multicolumn{4}{c}{ Área debajo de la curva } \\
\hline Pruebas & $\mathbf{4}$ años (15) & $\mathbf{5}$ años (17) & 6 años (18) \\
\hline Semejanzas y diferencias & .50 & .72 & .45 \\
Operaciones numéricas & $(.23-.76)$ & $(.45-.99)$ & $(.19-.72)$ \\
& .54 & .77 & .60 \\
Raven & $(.30-.78)$ & $(.54-.99)$ & $(.32-.87)$ \\
& .70 & .76 & .67 \\
& $(.47-.94)$ & $(.60-.91)$ & $(.45-.89)$ \\
\hline
\end{tabular}

Indicadores de validez

\begin{tabular}{|c|c|c|c|c|c|c|}
\hline Edades & Sensibilidad & Especificidad & $\mathbf{V p}$ & Vn & Fp & Fn \\
\hline 4 años & .76 & 1 & 19 & 5 & 0 & 6 \\
\hline 5 años & 1 & .28 & 43 & 2 & 5 & 0 \\
\hline 6 años & .87 & 1 & 41 & 3 & 0 & 6 \\
\hline Total & .66 & 103 & & 5 & & \\
\hline
\end{tabular}

Nota. Vp "Verdadero positivo", Vn "Verdadero negativo", Fp "Falso positivo" y Fn "Falso negativo"

Los valores del área debajo de la curva mostró a la prueba Raven como la más efectiva y estable en las edades para clasificar al niño positivo y negativo: 4 años (.70), 5 años (.76) y 6 años (.67) (Figura 1). Según los valores del área debajo de la curva el punto de corte propuesto puede ser útil para un propósito (Tabla 4). 

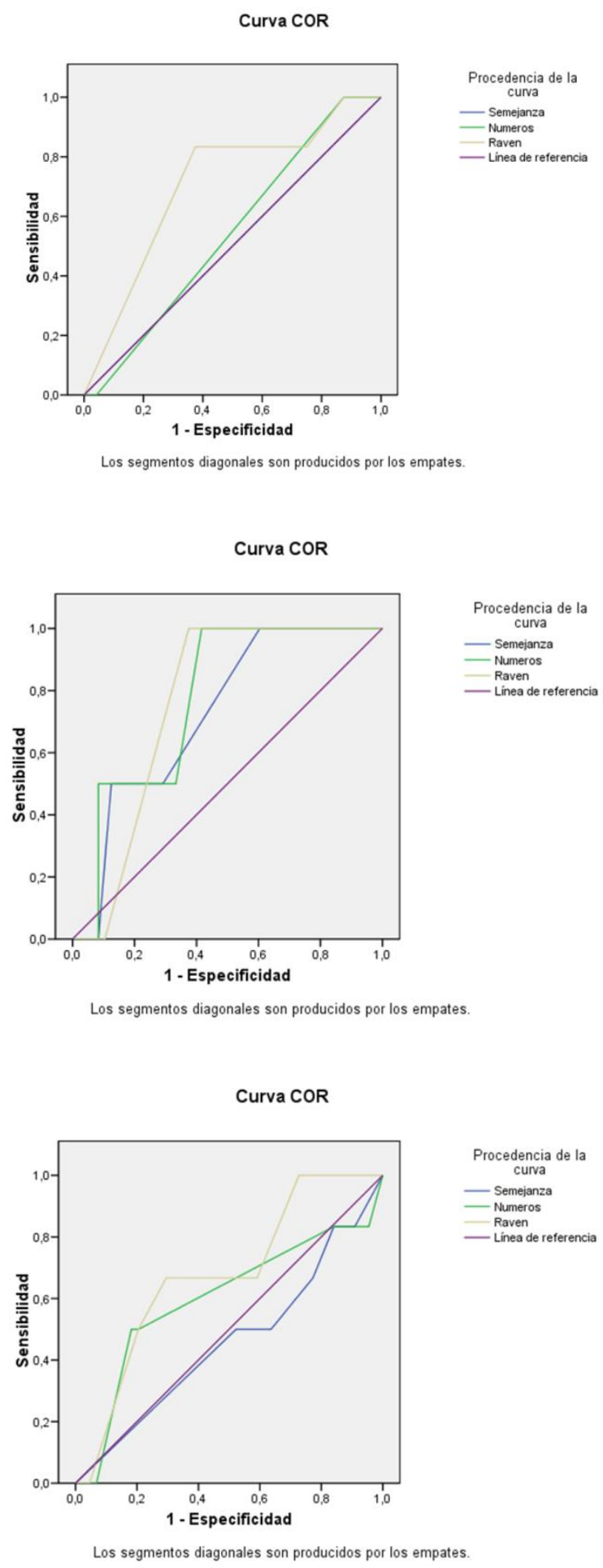

Figura 1. Área debajo de la curva de la prueba Raven y las tareas de razonamiento. (Primera 4 años, segunda 5 años y tercera 6 años) 


\section{DISCUSIÓN}

La prueba Raven es un instrumento con propiedades psicométricas apropiadas para detectar el riesgo intelectual en niños preescolares. Normalmente, los índices de confiabilidad reportados por los autores de pruebas de inteligencia se encuentran entre .70 y 95 (Fernández Liporace, Ongarato, Saavedra, \& Martina Casullo, 2004; Hazbún, Forno, Ivanovic, Durán, Castro, \& Ivanovic, 2003; Kamphaus, \& Reynolds, 2003). Según Ledesma, Molina y Valero (2002), valores superiores a .70 constituyen adecuados niveles de confiabilidad. En este sentido, el índice de confiabilidad de la prueba Raven resulta adecuada (.86).

En relación a la validez, la estrategia más frecuente es el cálculo de la correlación con una medida reconocida del constructo. Así, correlaciones positivas y significativas se interpretan como evidencias de una adecuada validez (Carretero-Dios \& Pérez, 2005; Muñiz, 2004). En este caso, la prueba Raven mostró correlaciones significativas con las tareas de razonamiento Semejanzas - Diferencias $\left(.70^{* *}\right)$ y Operaciones numéricas $\left(.69^{* *}\right)$.

Las altas correlaciones entre la prueba de pesquisa y una medida reconocida no son suficientes para dar criterios de validez. Resnick y Entin (2002) recomiendan, además de tener altas correlaciones, que ambas pruebas coincidan al clasificar el trastorno de interés de una forma limitada. De esta forma, el punto de corte propuesto por la investigación debe coincidir de forma adecuada con la norma mexicana al clasificar el niño positivo y negativo.

En este sentido, el $86 \%$ de los niños clasificados por la norma mexicana son igualmente clasificados por el punto de corte cubano. De 115 niños clasificados con riesgo intelectual por la norma mexicana fueron clasificados 103 niños $(89 \%)$ por el punto de corte cubano, con doce falsos negativos. De quince niños clasificados sin riesgo por la norma mexicana fueron clasificados diez niños $(66 \%)$ por el punto de corte cubano, con cinco falsos positivos. Los resultados indican la fortaleza del corte cubano para detectar el riesgo intelectual en el niño preescolar y en menor medida para descartar el niño escolar sin el trastorno. De ahí la importancia de la coincidencia como validez, pues indica el uso práctico del instrumento.

La fortaleza del corte cubano se puede observar en la mayoría de las edades 
evaluadas. A los 4 años la coincidencia es adecuada, el 86\% de los niños clasificados por la norma mexicana es clasificado, en iguales condiciones, por el corte cubano. A los 5 años, el $90 \%$ de los niños clasificados por la norma mexicana es clasificado, en iguales condiciones, por el corte cubano. A los 6 años, $88 \%$ de los niños clasificados por la norma mexicana es clasificado, en iguales condiciones, por el corte cubano.

En relación a la sensibilidad, el corte cubano clasifica mejor el riesgo intelectual en las edades 5 y 6 años ( 1 y .87 respectivamente), aunque a los 4 años es apropiada (.76) para detectar el riesgo intelectual.

En relación a la especificidad, el corte cubano clasifica mejor al niño sin trastorno en las edades 4 y 6 años. En estas edades, el 100\% de los niños sin riesgos fueron identificados por el corte cubano. Este es un elemento de gran importancia a tener en la práctica en estas edades, pues le ofrece al investigador la posibilidad de no utilizar otra prueba para descartar el trastorno. En la edad de 5 años, la especificidad es inadecuada, por tanto se debe tener cuidado al utilizar el corte cubano en esta edad, pues existe la posibilidad de evaluar de positivo al niño que no tiene el trastorno. Una posible solución a este problema es aplicar la prueba en una muestra mayor para obtener un punto de corte y clasificaciones mejor ajustadas.

En este sentido, el punto de corte es más efectivo en las edades de 4 y 6 años para detectar el riesgo intelectual y en menor medida con la edad de 6 años. De manera general, el instrumento Raven puede ser utilizado como prueba de pesquisa en la población cubana y utilizar el punto de corte que se propone en la presente investigación.

En conclusión, el instrumento de inteligencia Raven presenta propiedades psicométricas apropiadas para evaluar el rendimiento intelectual del niño preescolar. Además, el punto de corte propuesto es más efectivo en las edades 4 y 6 años.

\section{REFERENCIAS}

Barkl, S., Porter, A., \& Ginns, P. (2012). Cognitive training for children: effects on inductive reasoning, deductive reasoning and mathematics achievement in an Australian school setting. Psychology in the Schools, 49(9), 828-842.

Carretero-Dios, H., \& Pérez, C. (2005). Normas para el desarrollo y revisión de estudios instrumentales. International Journal of Clinical and Health Psychology, 5, 521-551.

Desco, M., Navas-Sanchez, F. J., Sanchez-González, J., Reig, S., Robles, O., Franco, C., ..., \& Arango, C. 
(2011). Mathematically gifted adolescents use more extensive and more bilateral areas of the frontoparietal network than controls during executive functioning and fluid reasoning tasks. Neuroimage, 57(1), 281-92.

Fernández Liporace, M., Ongarato, P., Saavedra, E., \& Martina Casullo, M. (2004). El Test de Matrices Progresivas, Escala General: un análisis psicométrico. Evaluar, 4, 50-69.

Hazbún, J., Forno, H., Ivanovic, D., Durán, M. C., Castro, C., \& Ivanovic, R. (2003). Estudio de la capacidad intelectual (Test de matrices progresivas de Raven) en escolares chilenos de 5 a 18 años.: I. Antecedentes generales, normas y recomendaciones. Iberpsicología: Revista Electrónica de la Federación Española de Asociaciones de Psicología, 8(1), 1579-4113.

Kamphaus, R. W., \& Reynolds, C. R. (2003). Reynolds intellectual screening test. Odessa, FL: PAR.

Ledesma, R., Molina, G., \& Valero, P. (2002). Análisis de consistencia interna mediante Alfa de Cronbach: un paquete basado en gráficos dinámicos. Psico-USF. 7(2), 143-152.

Leeuwen, M., Van den Berg, S., Hoekstra, R. A., \& Boomsma, D. (2007). Endophenotypes for intelligence in children and adolescents. Intelligence, 35, 369-380.

Luo, D., Thompson, L. A., \& Detterman, D. K. The criterion validity of tasks of basic cognitive processes. Intelligence, 34, 79-120.

Manga, D., \& Ramos, F. (2006). Luria Inicial: Evaluación neuropsicológica en la edad preescolar. Manual. Madrid: TEA Ediciones.

Manrique Millones, D., Flores-Mendoza, C., \& Millones Rivalles, R. (2015). Intelligence in Peru: Students' results in Raven and its relationship to SES. Intelligence, 51, 71-78.

Muñiz, J. (2004). La validación de los tests. Metodología de las Ciencias del Comportamiento, 5, 121-141.

Primi, R., Ferrão, M. E., \& Almeida, L. (2010). Fluid intelligence as a predictor of learning: A longitudinal multilevel approach applied to math. Learning and Individual Differences, 20, 446-451.

Raven, J. C. (2004). Test de matrices progresivas. Escala coloreada. Cuaderno de Matrices / Series A, AB y B. México: Paidós.

Resnick, R. J., \& Entin, A. D. (1971). Is an abbreviated form of the WISC valid for Afro-Americans? Journal of Consulting and Clinical Psychology, 36, 97-99.

Ritchie S. J., Bates, T. C., \& Plomin, R. (2015). Does Learning to Read Improve Intelligence? A Longitudinal Multivariate Analysis in Identical Twins from Age 7 to 16. Child Development, 86(1), 23-36.

Schlagenhauf, F., Rapp, M. A., Huys, Q. J., Beck, A., Wüstenberg, T., Deserno, L., .., \& Heinz, A. (2013). Ventral striatal prediction error signaling is associated with dopamine synthesis capacity and fluid intelligence. Human Brain Mapping, 34(6): 1490-1499.

Swets, J. A. (1988). Measuring the accuracy of diagnostic systems. Science, 240, 1285-1293.

Tong, F., \& Fu, T. (2013). Meta-Analysis of Fluid Intelligence Tests of Children from the Chinese Mainland with Learning Difficulties. PLoS ONE, 8(11), e78311. doi:10.1371/journal.pone.0078311

Van Bergen, E., de Jong, P. F., Maassen, B., Krikhaar, E., Plakas, A., \& Van der Leij, A. (2013). IQ of FourYear-Olds who go on to Develop Dyslexia. Journal of Learning Disabilities, 14, 1-10.

Yuan, Z., Qin, W., Wang, D., Jiang, T., Zhang, Y., \& Yu, C. (2012). The salience network contributes to an 
individual's fluid reasoning capacity. Behavioural Brain Research, 229(2), 384-90.

Zhao, X., Wang, Y. X., Liu, D. W., \& Zhou, R. L. (2011). Effect of updating training on fluid intelligence in children. Chinese Science Bulletin/ Psychology, 56(21), 2202-2205. 